\title{
THE APPEARANCE OF SPICULES IN HIGH RESOLUTION OBSERVATIONS OF Ca II H AND H $\alpha$
}

Tiago M. D. Pereira, Luc Rouppe van der Voort, and Mats Carlsson

Institute of Theoretical Astrophysics, University of Oslo, P.O. Box 1029 Blindern, NO-0315 Oslo, Norway; tiago.pereira@astro.uio.no Received 2016 March 8; accepted 2016 April 8; published 2016 June 14

\begin{abstract}
Solar spicules are chromospheric fibrils that appear everywhere on the Sun, yet their origin is not understood. Using high resolution observations of spicules obtained with the Swedish $1 \mathrm{~m}$ Solar Telescope, we aim to understand how spicules appear in filtergrams and Dopplergrams, how they compare in $\mathrm{Ca}$ II $\mathrm{H}$ and $\mathrm{H} \alpha$ filtergrams, and what can make them appear and disappear. We find that spicules display a rich and detailed spatial structure, and show a distribution of transverse velocities that, when aligned with the line of sight, can make them appear at different $\mathrm{H} \alpha$ wing positions. They become more abundant at positions closer to the line core, reflecting a distribution of Doppler shifts and widths. In $\mathrm{H} \alpha$ width maps they stand out as bright features both on disk and off limb, reflecting their large Doppler motions and possibly higher temperatures than in the typical H $\alpha$ formation region. Spicule lifetimes measured from narrowband images at only a few positions will be an underestimate because Doppler shifts can make them disappear prematurely from such images; for such cases, width maps are a more robust tool. In $\mathrm{H} \alpha$ and $\mathrm{Ca}$ II $\mathrm{H}$ filtergrams, off-limb spicules essentially have the same properties, appearance, and evolution. We find that the sudden appearance of spicules can be explained by Doppler shifts from their transverse motions, and does not require other convoluted explanations.
\end{abstract}

Key words: Sun: atmosphere - Sun: chromosphere

Supporting material: animation

\section{INTRODUCTION}

Spicules and fibrils are observed all over the Sun in chromospheric lines. Their very existence and transient, fast motions have been a challenge to explain. Much work has been carried out on this subject, with early reviews by Beckers (1968, 1972) and later reviews by Sterling (2000), Rutten (2012), and Tsiropoula et al. (2012). Some of the most pressing questions about spicules are (1) what drives them and (2) what is their contribution to the transfer of energy and mass from the photosphere to the corona.

When coined by Roberts (1945), the term "spicules" applied strictly to objects outside the solar limb. Since then, many objects that are believed to be their disk counterparts have been observed on the solar disk, resulting in a profusion of different terms used to refer to (mostly) the same objects (Beckers 1968; Grossmann-Doerth \& Schmidt 1992; Tsiropoula et al. 1994; Rutten 2006; Langangen et al. 2008; Judge et al. 2012; Sekse et al. 2013b). Here we adopt the term "spicules" for both limb and disk objects, clarifying whether they are "off limb" or "on disk" when necessary.

What is the allure of spicules? What makes them a worthwhile research topic? For one, they appear nearly everywhere on the Sun and are dominant in some chromospheric filtergrams such as $\mathrm{Ca}$ II $\mathrm{H}$. Physically resembling jets, rooted in the photosphere, and reaching coronal heights, from early on it was natural to assume that they may contribute toward heating the corona and supplying it with mass. Indeed early estimates indicated that the upward mass flux of spicules can be 100 times larger than the solar wind (Pneuman \& Kopp 1977, 1978); even if most of that flux comes back down, if only a few a few percent continue upward, it is enough to drive the solar wind.

Much of the research on spicules has followed from advances in observations. Early hopes for their importance in coronal heating were dashed when no spicular emission was observed in coronal lines (Withbroe 1983). Interest was rekindled when Hinode observations revealed that some spicules (so-called type II) were more violent than previously thought, fading from the $\mathrm{Ca}$ II $\mathrm{H}$ passband and not seen to fall back down (De Pontieu et al. 2007). While Zhang et al. (2012) questioned the existence of two types of spicules, Pereira et al. (2012) analyzed some of the same data sets and concluded otherwise, finding that type II are the dominant type. The fading of $\mathrm{Ca}$ II $\mathrm{H}$ spicules has been linked to higher energy emission from the transition region and corona (De Pontieu et al. 2009), and the advent of the Interface Region Imaging Spectrograph (IRIS, De Pontieu et al. 2014) has shown that spicules have a clear signal in transition region filters and they continue to evolve in higher temperatures after fading from $\mathrm{Ca}$ II $\mathrm{H}$ (Pereira et al. 2014). Judge \& Carlsson (2010) suggest that despite their dominance in chromospheric images, spicules make up less than $1 \%$ of the mass of the whole chromosphere. Judge et al. (2011) speculate that spicules may not be real mass motions, but optical illusions arising from warped magnetic sheets, a view that Judge et al. (2012) claim to find observational evidence for. All of these findings highlight the fact that the understanding and modeling of spicules relies on their observed properties, some of which are still contested.

This work aims to contribute to the discussion of the observed properties of spicules, in particular how different observations relate to one another and how to properly trace the histories of spicules. To achieve those aims, we make use of a unique set of observations, which is introduced in Section 2. In Section 3 we compare spicules in $\mathrm{Ca}$ II $\mathrm{H}$ and $\mathrm{H} \alpha$, and in Section 4 we discuss their appearance in filtergrams and Dopplergrams. We discuss our findings in Section 5 and finish with a summary of our results in Section 6. 


\section{OBSERVATIONS}

We obtained a series of observations at the Swedish $1 \mathrm{~m}$ Solar Telescope (SST; Scharmer et al. 2003) on La Palma using the Crisp Imaging SpectroPolarimeter (CRISP; Scharmer et al. 2008) instrument. CRISP is a dual Fabry-Pérot interferometer (FPI) that contains three high-speed CCD cameras ( 35 frames s${ }^{-1}$ with an exposure time of $17 \mathrm{~ms}$ per frame): two cameras behind the FPI and a polarizing beam splitter, and a third "wide band" camera located before the FPI, which is used as an anchor channel for image processing. The CRISP field of view is approximately $61^{\prime \prime} \times 61^{\prime \prime}$, with a plate scale of $0{ }^{\prime \prime} 058 \mathrm{pixel}^{-1}$. For this work we used a CRISP configuration scanning only the $\mathrm{H} \alpha$ line: using a pre-filter with a full width at half maximum (FWHM) of $0.49 \mathrm{~nm}$ we scanned the line along 25 positions, from -0.12 to $0.12 \mathrm{~nm}$ around the line core in $0.01 \mathrm{~nm}$ steps. The FPI allows for very fast wavelength tuning $(<50 \mathrm{~ms})$ within a spectral line; the cadence of our setup was $5.5 \mathrm{~s}$. In addition to CRISP, we used the so-called "blue tower" at the SST (see, e.g., Henriques 2012). Using a dichroic beam splitter, the blue part of the spectrum $(\lambda<500 \mathrm{~nm})$ was channeled to two additional imaging cameras: one was placed behind a Ca II $\mathrm{H}$ interference filter (FWHM of $0.11 \mathrm{~nm}$, centered at $396.88 \mathrm{~nm}$ ) and the other behind a wide band filter (FWHM of $1 \mathrm{~nm}$ ) centered at $395.37 \mathrm{~nm}$ (the pseudo-continuum between the $\mathrm{Ca}$ II $\mathrm{H}$ and $\mathrm{Ca}$ II $\mathrm{K}$ lines).

The observations took place on 2014 June 17; the time sequence analyzed here was obtained between 10:20 UT and 11:15 UT. For this period the atmospheric conditions on La Palma resulted in excellent, stable seeing. The target was quiet Sun at the solar north pole, centered at solar $(x, y)$ coordinates of $24^{\prime \prime}, 939^{\prime \prime}$. In Figure 1 we show the target in context with images from the Atmospheric Imaging Assembly (AIA; Lemen et al. 2012) in the 30.4 and $19.3 \mathrm{~nm}$ channels. As can be seen in the $19.3 \mathrm{~nm}$ images, there was no polar coronal hole during this period.

The CRISP data were reduced using the CRISPRED pipeline (de la Cruz Rodríguez et al. 2015). We made use of the MultiObject, Multi-Frame Blind Deconvolution (MOMFBD) image restoration technique of van Noort et al. (2005), and employed the cross-correlation method of Henriques (2012) to minimize the seeing deformations introduced by the non-simultaneity of the narrowband CRISP images. The blue images were flat fielded and dark subtracted, and were also restored using MOMFBD. The wide band cameras of CRISP and the blue beam were used to co-align both series.

To enhance the visibity of spicules, we employed radial density filters (see discussion in Skogsrud et al. 2015, and references therein). These filtered images are built by dividing the images by a mean intensity profile as a function distance to the limb. For the CRISP images shown, we applied radial filters for each wavelength independently. Unless otherwise noted, all images shown here have been radially filtered. Following on the discussion of Skogsrud et al. (2015) we made sure that the radial filters left no spicular signal under the noise.

To visualize, connect, and interpret the data we made extensive use of CRISPEX (Vissers \& Rouppe van der Voort 2012).

\section{COMPARING $\mathrm{H} \alpha$ WITH CA II H SPICULES}

While spicules have been observed in many chromospheric lines, most studies in the early literature (pre-Hinode) made use of $\mathrm{H} \alpha$ observations (see Beckers 1968, 1972). The advent of Hinode, with SOT's Broadband Filter Imager (BFI) highquality $\mathrm{Ca}$ II $\mathrm{H}$ filtergrams led to renewed interest in spicules and the discovery of a widespread new dynamic behavior (e.g., De Pontieu et al. 2007; Pereira et al. 2012). When confronting the results from Hinode's $\mathrm{Ca}$ II $\mathrm{H}$ spicules with earlier literature, one can ask why the more dynamic behavior of type II spicules was not observed in earlier $\mathrm{H} \alpha$ observations. Pereira et al. (2013) showed that by just degrading the Hinode data to similar conditions of earlier observations, one can derive the properties of classical spicules. The authors also compared SOT BFI Ca II $\mathrm{H}$ filtergrams with NFI $\mathrm{H} \alpha$ wing filtergrams and concluded that the spicules were very similar in both lines. Nevertheless, slight differences in the spicules were caused by comparing a composite $\mathrm{H} \alpha \pm 80 \mathrm{pm}$ wing filtergram with a broader filter in Ca II H. Such differences could, in an extreme scenario, still cause doubt on whether the evolution of spicules would be the same in both spectral lines.

Here we compare $\mathrm{Ca}$ II $\mathrm{H}$ filtergrams with synthetic $\mathrm{H} \alpha$ filtergrams computed from the CRISP spectra. In Figure 2 we show a comparison between the $\mathrm{Ca}$ II $\mathrm{H}$ and $\mathrm{H} \alpha$ filtergrams for different regions and times. The $\mathrm{H} \alpha$ filtergrams were calculated by convolving the CRISP spectral images with a Gaussian transmission function with a FWHM of $0.1 \mathrm{~nm}$ (the choice of FWHM is discussed in the next paragraph). The $\mathrm{Ca}$ II $\mathrm{H}$ images shown suffer from fringing, which is an observational artifact that was not possible to remove in post-processing because of its time dependency. These fringes show up clearly as regular circular or linear patterns, are enhanced by the radial filtering, and are noticeably different from the underlying spicules.

A $0.1 \mathrm{~nm} \mathrm{H} \alpha$ filtergram still shows a moderate amount of opaque material just above the limb, with individual spicules difficult to discern. On the $\mathrm{Ca}$ II $\mathrm{H}$ filtergrams, the individual spicules can be traced all the way to the limb. The reason for this apparent discrepancy is that for the same distance near the line core, $\mathrm{H} \alpha$ images still have a considerable amount of chromospheric contribution, while in $\mathrm{Ca}$ II $\mathrm{H}$ the wings are progressively formed much closer to the photosphere. Using a wider filter for the $\mathrm{H} \alpha$ filtergrams, one can obtain nearly identical results. Our $\mathrm{H} \alpha$ wavelength window is only $0.24 \mathrm{~nm}$, and therefore for widths larger than $0.1 \mathrm{~nm}$ our synthetic filtergrams cannot sample the far wings of $\mathrm{H} \alpha$. Nevertheless, we estimate that with a FWHM of $0.3 \mathrm{~nm}$ the $\mathrm{H} \alpha$ filtergrams will look closest to the $0.11 \mathrm{~nm}$ Ca II $\mathrm{H}$ filtergrams.

Figure 2 shows a remarkable similarity between the $\mathrm{H} \alpha$ and $\mathrm{Ca}$ II $\mathrm{H}$ spicules. Using arrows as a visual aid, we note several details of fine structure and spicule length. Aside from small differences in intensity and noise levels (and the fuzzier bottom half due to the $\mathrm{H} \alpha$ filter), we find that the spicule shapes, extent, and lifetimes are essentially identical between $\mathrm{H} \alpha$ and $\mathrm{Ca}$ II $\mathrm{H}$. In this quiet Sun region most of the spicules are of type II, fading at around their maximum extent. The same is observed in $\mathrm{H} \alpha$. Long sequence movies show the same evolution in both filters.

\section{SPICULES AND FIBRILS IN FILTERGRAMS AND DOPPLERGRAMS}

\subsection{Off limb and On disk}

Spicules have been traditionally observed in $\mathrm{H} \alpha$ and $\mathrm{Ca}$ II $\mathrm{H}$ filtergrams (as shown above, the differences in spicule properties between both lines are negligible). Both broad and 

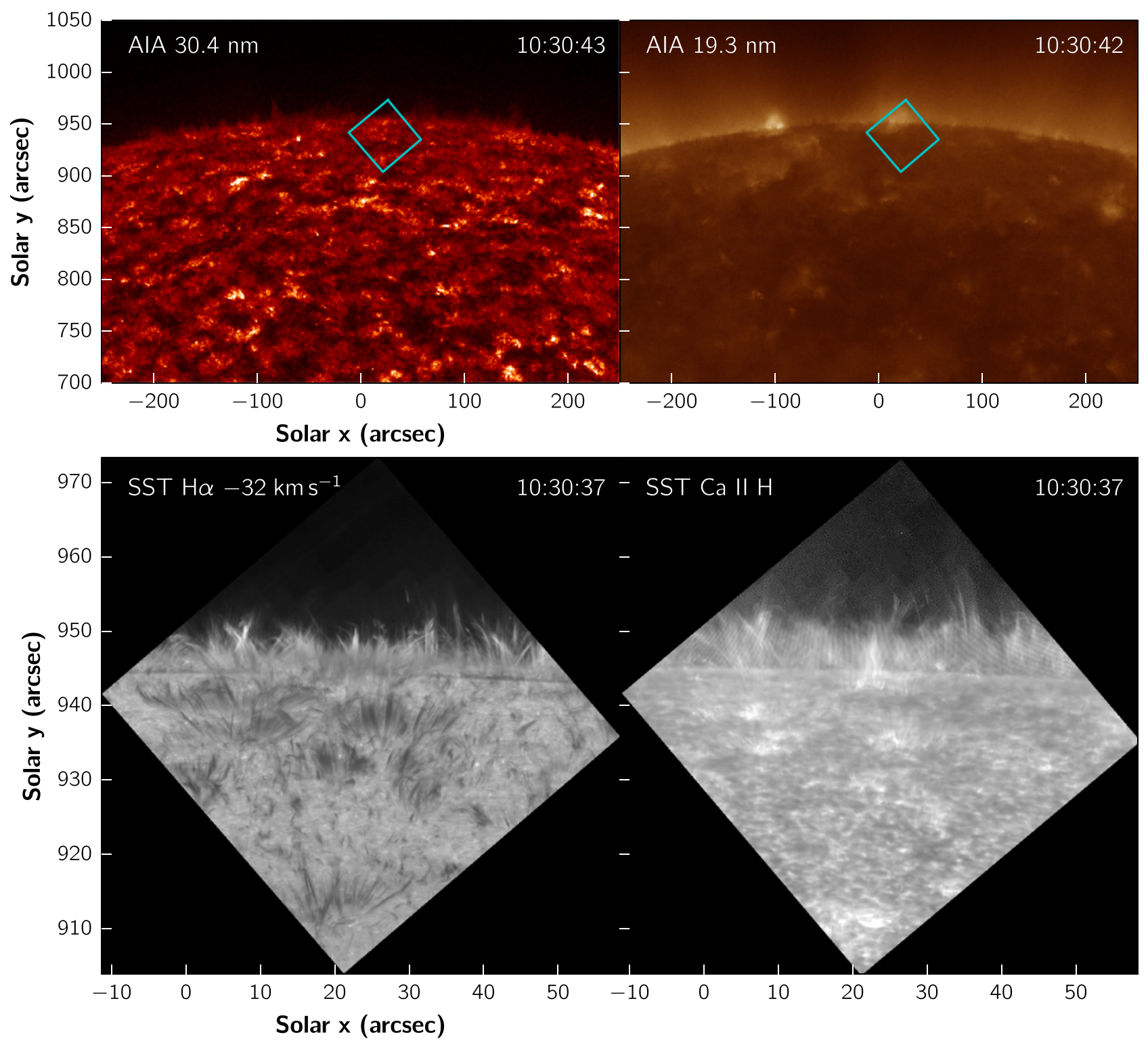

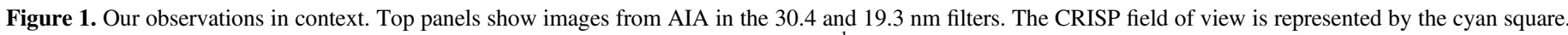

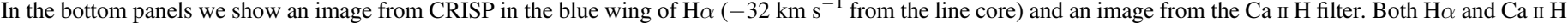
have been radially filtered to enhance the visibility of spicules (see the text). The observing time (in UT) of each image is printed in the top right corners.

narrow filtergrams centered in the cores of chromospheric lines show little evidence of spicules on the solar disk-hence they were classified earlier as limb objects. With broad filters the spicule signal is very low compared with the signal of photospheric light present in the line wings (Carlsson et al. 2007; Beck et al. 2013). With narrow filters individual spicules are usually indistinguishable from the crowded canopy of chromospheric fibrils. But spicules have clear spectral signatures in the form of Doppler-shifted, wider line profiles with increased absorption in the red or blue wings. Such signatures have been observed for spicules on disk (e.g., Langangen et al. 2008; Rouppe van der Voort et al. 2009; Sekse et al. 2013b; Yurchyshyn et al. 2013; Kuridze et al. 2015) and off limb (Pereira et al. 2014).

In Figure 3 we show the same field of view in different $\mathrm{H} \alpha$ images. In the top panel we show narrowband CRISP images at wavelengths from -50 to $50 \mathrm{~km} \mathrm{~s}^{-1}$ from the line center. At $\pm 50 \mathrm{~km} \mathrm{~s}^{-1}$ one can see the limb spicules very clearly, while only a faint hint of the disk spicules is visible. This is likely because fainter events on disk will be obscured by the photospheric light background, and a radial filter was applied on the limb spicules. As one looks closer to the line core, the spicules become more abundant and their bundling as "bushes" rooted in the network becomes more obvious.

In the bottom panel of Figure 3 we show different composite quantities: five Dopplergrams, a synthetic filtergram, and a map of normalized $\mathrm{H} \alpha$ widths. The Dopplergrams were built by taking the difference of two CRISP narrowband images at symmetric positions from the line center. Such difference images highlight regions with strong Doppler shifts. On disk, with $\mathrm{H} \alpha$ in absorption, negative velocities (blueshifts) appear black, while positive velocities (redshifts) appear white. Off limb, when $\mathrm{H} \alpha$ is in emission, the reverse is true: blueshifts appear white, while redshifts appear black. When a feature appears in a Dopplergram at a particular velocity, it does not mean that it has a flow at that velocity—instead it means that its 

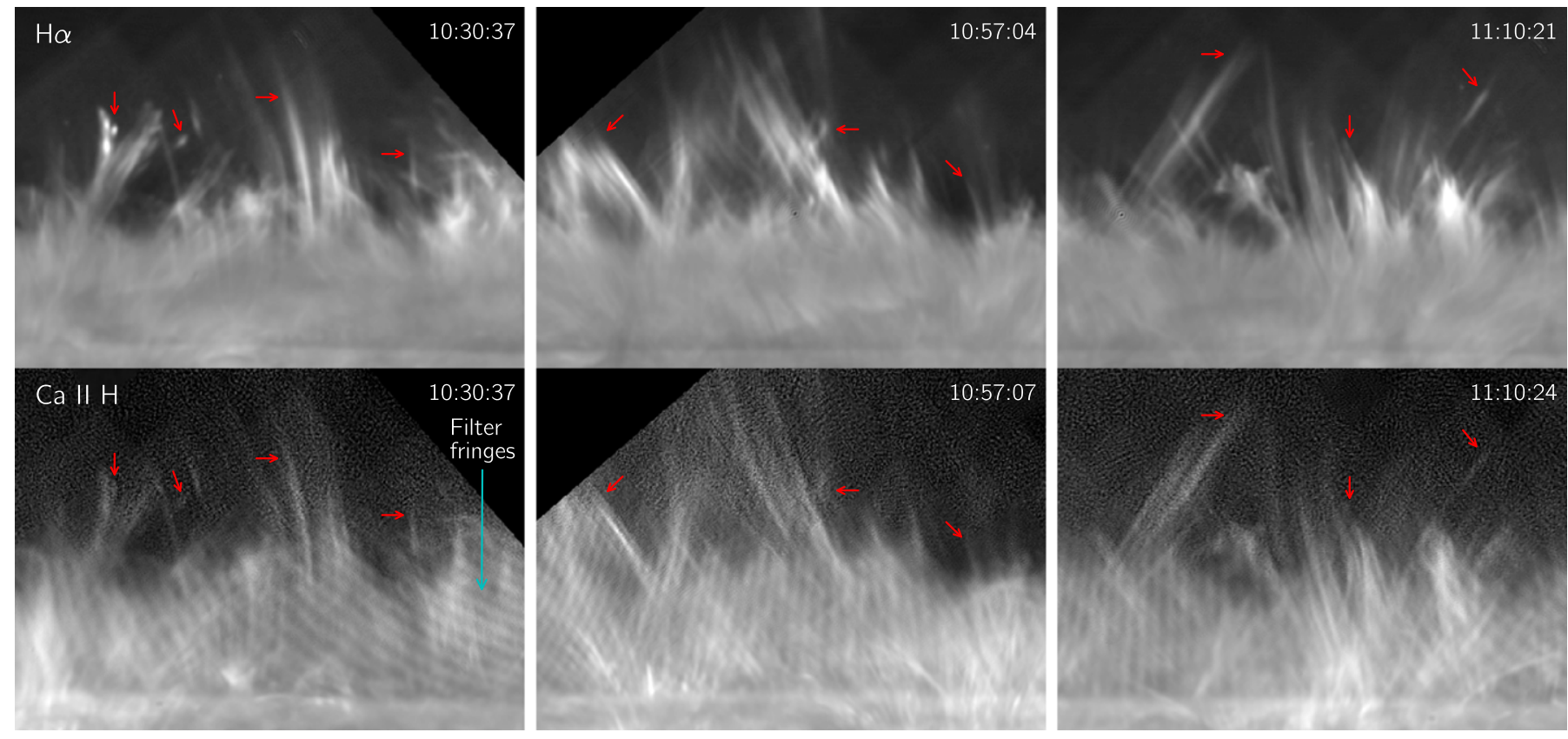

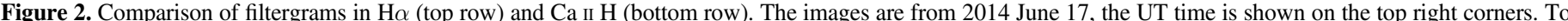

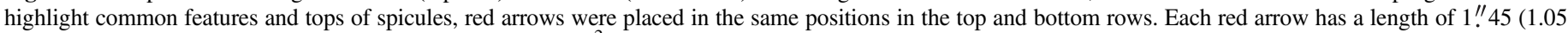

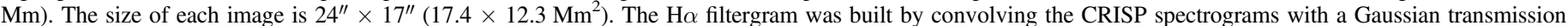
function, using a FWHM of $0.1 \mathrm{~nm}$. The Ca II $\mathrm{H}$ filtergram has a FWHM of $0.11 \mathrm{~nm}$.

line profile is Doppler shifted enough or wide enough so that some intensity decrease/increase takes place at that wavelength (see discussion in Lipartito et al. 2014). Spicules stand out in such Dopplergrams, which eliminate most of the photospheric background and show in great spatial detail their predominant line of sight motions. The abundances of spicules at different Dopplergrams reflect their velocity distribution (of the three kinds of motions: upflow, transverse, and torsional; see Sekse et al. 2013b). In the far wing images one sees a lot less events, presumably the extreme tail of the motion distribution. However, as one moves farther from the limb, the $\mathrm{H} \alpha$ profile also gets naturally narrower. Therefore, the same velocity shift that would cause a disk spicule to be visible in the far wing Dopplergrams will not necessarily make it visible in the same Dopplergram above the limb. Hence we often see longer limb spicules at wavelengths closer to the line core (both in images and Dopplergrams). To compensate for this and increase the visibility of spicules in Dopplergrams, we created a "composite Dopplergram" by combining two Dopplergrams made of radially filtered images: one at $\pm 50 \mathrm{~km} \mathrm{~s}^{-1}( \pm 0.11 \mathrm{~nm})$ and the other at $\pm 5 \mathrm{~km} \mathrm{~s}^{-1}( \pm 0.01 \mathrm{~nm})$, also shown in Figure 3 . Both Dopplergrams were multiplied by a step function with a smooth profile: the lower part of the image is the $\pm 50 \mathrm{~km} \mathrm{~s}^{-1}$ Dopplergram and the upper part is the $\pm 5 \mathrm{~km} \mathrm{~s}^{-1}$ Dopplergram; the dashed line is the middle point where they are blended. Thus the combined Dopplergrams are a "cleaner" way to visualize spicules from the limb to their very top, mostly avoiding the chromospheric "haze" that is prominent when looking just above the limb at wavelengths close to the line core.

From the synthetic filtergram one can see the advantage of broadband images over narrowband images: they capture signals from spicules on both red and blue wings. Despite having a relatively narrow $(0.1 \mathrm{~nm})$ FWHM, the synthetic filtergram still shows disk spicules as very faint and difficult to identify, and the considerable opacity of chromospheric fibrils also makes it difficult to trace the lower part of limb spicules. Broader filters make the identification of limb spicules easier, but make limb spicules even fainter (e.g., Hinode SOT BFI's Ca II $\mathrm{H}$ filtergrams).

One key diagnostic in Figure 3 is the normalized $\mathrm{H} \alpha$ width. For each pixel, the width $W$ was calculated as the FWHM of the line profile. On the disk the line is in absorption, so this is well defined. For locations more than $\approx 3 \mathrm{Mm}$ above the limb, the line is in pure emission, so the FWHM is also well defined. However, for regions in the first $\approx 3 \mathrm{Mm}$ above the limb it becomes problematic to define a FWHM because the line profiles are transitioning from absorption to emission and will have a variety of shapes in between. In such cases the emission begins as peaks on the far blue and red wings, which become bigger away from the limb, going to an absorption peak with a central reversal peak and finally pure emission. Measuring $W$ as FWHM in those intermediate profiles is often meaningless (e.g., when there is emission in the wings but an absorption core still deeper than the far wings, half of the maximum is an arbitrary location). Our approach to measuring $W$ in all locations was to start on the outside edges of the wings and move inward toward the line core, finding the wavelength difference between the first positions where the intensity equals half of the maximum. This minimizes only some of the issues in the intermediate limb region. Because our CRISP coverage only goes $\pm 0.12 \mathrm{~nm}$ around the line core, the very far wings of $\mathrm{H} \alpha$ are not covered and the maximum intensity of the spectra lies in these far wings and not the continuum. To compensate for all these limitations, we normalize the $W$ by $\bar{W}(\mu)$ (i.e., the width of the mean spectrum at the same distance from the limb), which was calculated by averaging the complete time series along radial curves parallel to the limb. Therefore the quantity plotted in Figure 3 can be seen as a "width enhancement." It is also visible that in the critical region up 

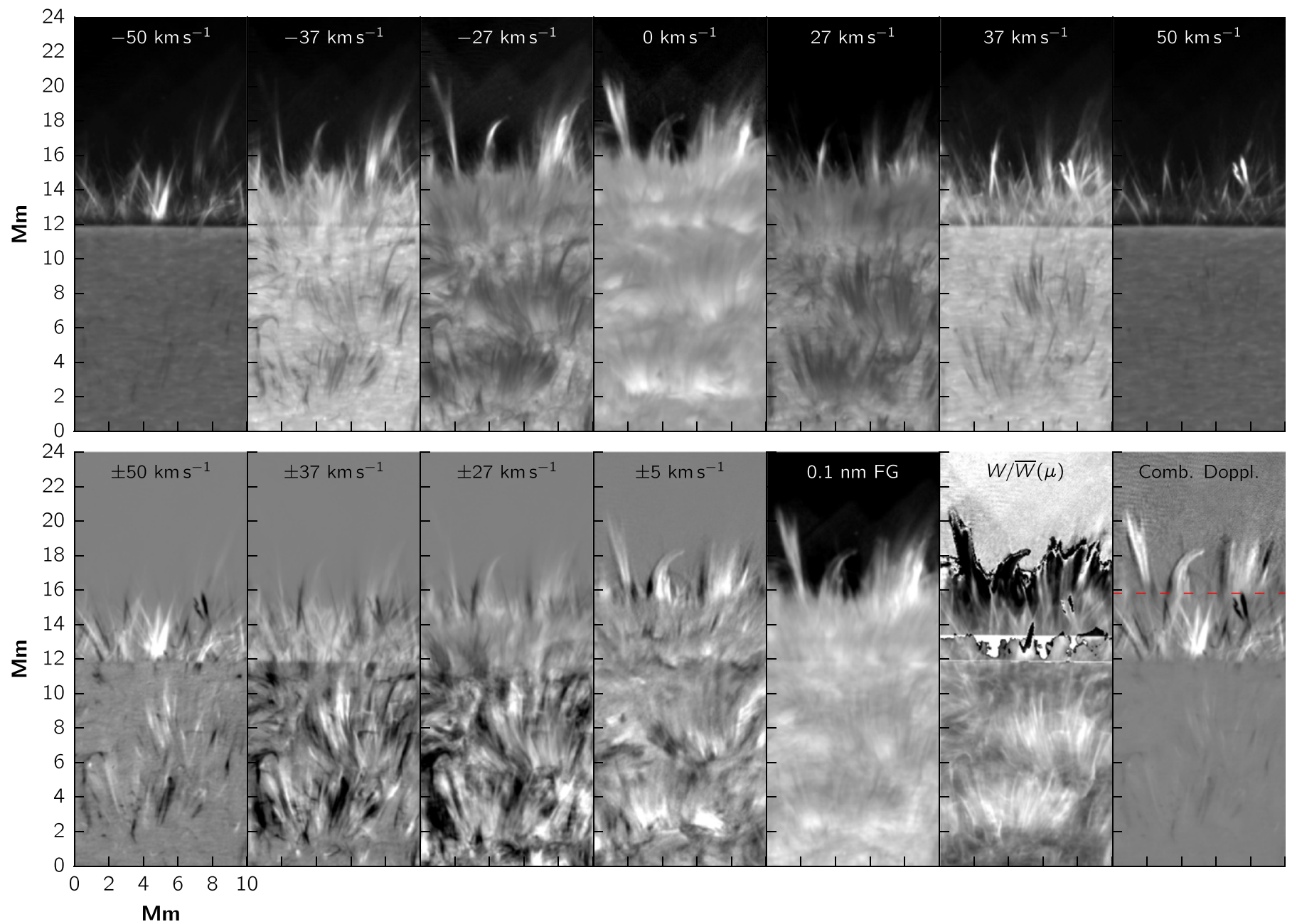

Figure 3. $\mathrm{H} \alpha$ observations and properties of spicules and fibrils observed on 2014 June 17, 11:43 UT. Top panels: H $\alpha$ intensities at different positions in the line profile, from $-50 \mathrm{~km} \mathrm{~s}^{-1}$ to $50 \mathrm{~km} \mathrm{~s}^{-1}$ (labels shown at the top), all radially filtered to enhance spicule visibility. Bottom panels: from left to right, with labels shown at the top: Dopplergrams at different distances from \pm 50 to $\pm 5 \mathrm{~km} \mathrm{~s}^{-1}$, filtergram with $0.1 \mathrm{~nm}$ Gaussian FWHM, H $\alpha$ width divided by the mean width at each $\mu$ value, and combined Dopplergram (see the text for details). Intensities in all panels were individually scaled for improved visibility and contrast.

(An animation of this figure is available.)

to $\lesssim 1.7 \mathrm{Mm}$ above the limb there are many cases where the width maps are very irregular — such regions should be ignored. Just above the spicules the width maps in Figure 3 appear white, meaning large width. This effect comes from the noisethe real signal being so weak that our approach mistakes noise for the signal and finds large widths. We chose not to mask these areas because these white areas above the spicules are an indicator of no spicule signal. The black areas just underneath, despite showing very low widths, are an indicator that there is still an emission profile and a little spicule signal is left (e.g., the large spicule at $(x, y) \approx(2,18) \mathrm{Mm}$ appears prominently in near core images and Dopplergrams, but the emission line profile is rather narrow, so it appears mostly as dark in the width map).

By taking the normalized width maps, we put the limb spicules on a common (width enhancement) scale as the disk spicules. They both appear, very noticeably, as width enhancements. We find numerous spicules in bushes rooted in the network, and they stand out even more from the background than in Dopplergrams. As in broadband filtergrams, width maps allow one to see both redshifted and blueshifted spicules, but with the huge advantage of being sensitive to both spicules on the disk and above the limb (notwithstanding the issues at the limb noted above). Crucially, width maps are much more reliable at tracing the full lifetime and length of spicules, which can prematurely disappear from Dopplergrams and filtergrams.

\subsection{Appearance and Disappearance}

The idea of spicules as jets of chromospheric material is as old as the term "spicules" itself (Roberts 1945). From early observations (e.g., Roberts 1945; Lippincott 1957) the early life of spicules was observed as an apparent upward motion. This view has so far stood the test of time: most studies agree that spicules show an upward motion in the early phase of their lives (e.g., Beckers 1968, 1972; Nishikawa 1988; Suematsu et al. 1995; De Pontieu et al. 2004; Tsiropoula et al. 2012). What happens in the later stages was unclear early on (Beckers 1968), and from Hinode Ca II $\mathrm{H}$ filtergrams it appears that spicules can be divided into two distinct groups (De Pontieu et al. 2007): type I spicules show a rise and fall, while type II spicules seem more violent and simply fade from $\mathrm{Ca}$ II $\mathrm{H}$ 
filtergrams with no downward motion observed (type II spicules dominate in quiet Sun and coronal holes; see Pereira et al. 2012). Analyses using IRIS data (Pereira et al. 2014; Skogsrud et al. 2015) show that after fading from $\mathrm{Ca}$ II $\mathrm{H}$ filtergrams, spicules continue to evolve and show a downward phase in filtergrams sampling higher temperatures, suggesting rapid heating.

Despite mounting evidence pointing toward spicules as ejections of material, some observational properties remain difficult to reconcile with this view. Perhaps most important, the complete time evolution is observed for only a small number of them. In movies many of the spicules simply appear around their maximum length, with no clear observation of their rise or when they started. The swaying transverse motion of spicules in their dense bushes is a likely cause. The large spatial superposition of spicules, particularly at the limb, makes it challenging to track every individual spicule (e.g., see "Selection Effects and Errors" in Pereira et al. 2012). Spicules also recur frequently from the same footpoint region (Beckers 1968; Suematsu et al. 1995; De Pontieu et al. 2011; Pereira et al. 2012; Sekse et al. 2013b; Yurchyshyn et al. 2013), meaning that when a particular spicule disappears it can be quickly replaced by another of similar intensity in the same place. Judge et al. (2012; hereafter JCR12) study disk spicules at a wavelength of $\mathrm{H} \alpha+0.11 \mathrm{~nm}$, and find spicules that appear and disappear suddenly over several $\mathrm{Mm}$. The authors claim this is evidence that spicules are not jets but instead an optical superposition caused by sheets of chromospheric material, a view earlier postulated by Judge et al. (2011). Our analysis is at odds with this interpretation.

We analyze a few cases of the so-called "suddenly appearing spicules" found by JCR12. In our data set those events seem rather rare: visual inspection finds only a handful of clear cases in the whole time series, and certainly a lot less than the 1/ 3-1/8 estimate of Lipartito et al. (2014, hereafter LJKG14). Here we note that the observations used by LJKG14 and JCR12 were from an active region while we observe the quiet Sun. In Figure 4 we illustrate the time evolution of one of these events. In the top row we show CRISP images at $+50 \mathrm{~km} \mathrm{~s}^{-1}$ or $+0.11 \mathrm{~nm}$ away from the $\mathrm{H} \alpha$ core (red wing), which is the same wavelength used by JCR12. In the second row we show the $\mathrm{H} \alpha+41 \mathrm{~km} \mathrm{~s}^{-1}(+0.1 \mathrm{~nm})$ intensity and the normalized width $W / \bar{W}(\mu)$. On the bottom panel we show spectrograms along the spicule's axis (shown as a dashed line in the upper three rows). The spectrograms were divided by the mean spectrum as a function of viewing angle, which as noted by LJKG14 makes it easier for spicules to stand out: they appear as dark bands the line wings. The normalized width (scale on top, from 0.9 to 1.25 ) along the spicule axis is overplotted on the same panel. At $t \approx 22.1 \mathrm{~s}$ a spicule appears seemingly along its whole length at $+50 \mathrm{~km} \mathrm{~s}^{-1}$, and is visible until at least $t \approx 60.8 \mathrm{~s}$. At $+41 \mathrm{~km} \mathrm{~s}^{-1}$ the spicule appears sooner, at $t \approx 11.1 \mathrm{~s}$, and is visible for at least a frame longer than at $+50 \mathrm{~km} \mathrm{~s}^{-1}$. Finally, in the width images it is clear that a structure is already present before it is visible in any of the red wing images, and already at the start of the sequence shown (unfortunately, for previous times it is not easy to follow this spicule's evolution in the width maps). On the spectrograms one can see how the width and Doppler shift of the feature evolve. In the first frame one can see a slightly increased width, $W / \bar{W}(\mu) \approx 1.05$ in the first $\mathrm{Mm}$ of the structure. The normalized line profile shows a darkening in both wings. As time evolves there is a clear increase in width and redshift. When the spicule appears at $+50 \mathrm{~km} \mathrm{~s}^{-1}, W / W(\mu) \approx 1.15$, and a redshift is evident by the red wing being very dark and the blue wing being lighter and even appearing as white (meaning its intensity is above the mean spectrum intensity). At the peak of strongest wing absorption, the spicule has a width enhancement of about 1.25, which later lowers as it evolves. In the last frames the redshift along the spicule is still visible, a hint that there could be different mechanisms for the width enhancement and transverse motions. The example shown in Figure 4 is not atypical; all the suddenly appearing spicules we analyzed were visible in width maps before appearing at $\mathrm{H} \alpha+$ $50 \mathrm{~km} \mathrm{~s}^{-1}$ (but again, we stress that in our data set these events were rare).

Figure 5 shows a different example of suddenly appearing spicules, now at the limb. We compare $\mathrm{H} \alpha$ wing intensities at $\pm 23 \mathrm{~km} \mathrm{~s}^{-1}( \pm 0.05 \mathrm{~nm})$ from the line center with a combined Dopplergram (see above) and a synthetic filtergram with a FWHM of $0.1 \mathrm{~nm}$. For compactness and the sake of clarity, the first frame already shows an intermediate stage in the life of the spicule, and only every second observed frame is shown. In the blue wing images at $-23 \mathrm{~km} \mathrm{~s}^{-1}$ we see a large spicule rising about $\approx 8 \mathrm{Mm}$ above the limb (and above the fibril canopy at $\approx 5 \mathrm{Mm}$ ). The spicule has multiple strands or threads. In the first frame its top half is noticeably blueshifted. From the combined Dopplergram, especially at later stages, one can find what looks like the bottom part of the spicule (or at least a structure that is aligned with what one would expect to be bottom part of the spicule); the bottom part is also multistranded and shows an increasing redshift: negligible in the first frame, then slowly increasing until $t \approx 55.3 \mathrm{~s}$. Seen in the blue wing, the top of the spicule starts getting fainter until it quickly disappears at $t \approx 44.3 \mathrm{~s}$. On the other hand, the same structure gets progressively brighter in the red wing images, because it is hardly visible in the first frame and reaching maximum brightness at around $t \approx 60 \mathrm{~s}$. After $t \approx 66.4 \mathrm{~s}$ the spicule starts getting fainter in the red wing and appears quickly in the blue wing images. This appearance and disappearance from the wing images is a consequence of line of sight velocity shifts from the swaying motion of the spicule. This is made clearer in the Dopplergrams, where the top of the spicule quickly goes from white (blueshifted) to black (redshifted) and again to white. Different strands of the spicule also appear to be moving with different velocities. Sensitive to both wings of the line, the filtergram intensities show a rather consistent spicule intensity with no sudden appearances or disappearances.

The example in Figure 5 is a rare occurrence. For such a scenario to be clearly observed, the spicule has to be taller than most and with a transverse motion closely aligned toward the observer (for shorter spicules there is too much superposition). Nevertheless, this example highlights the limitations of narrowband images for studying the lives of spicules. By only looking at a particular wavelength one can miss part of the evolution, assume that the spicule appears or disappears suddenly, and confuse different stages of the same event as distinct, recurring events.

\section{DISCUSSION}

We showed how spicules appear in different positions in $\mathrm{H} \alpha$, in filtergrams and Dopplergrams. Comparing limb spicules in $\mathrm{Ca}$ II $\mathrm{H}$ and $\mathrm{H} \alpha$ filtergrams we find that there is very little difference between the two filters. The same fine scale and 
$5.5 \mathrm{~s} 11.1 \mathrm{~s} 16.6 \mathrm{~s} 22.1 \mathrm{~s} 27.7 \mathrm{~s} 33.2 \mathrm{~s} 38.7 \mathrm{~s} 44.3 \mathrm{~s} 49.8 \mathrm{~s} 55.3 \mathrm{~s} 60.8 \mathrm{~s} 66.4 \mathrm{~s} 71.9 \mathrm{~s} 77.4 \mathrm{~s}$
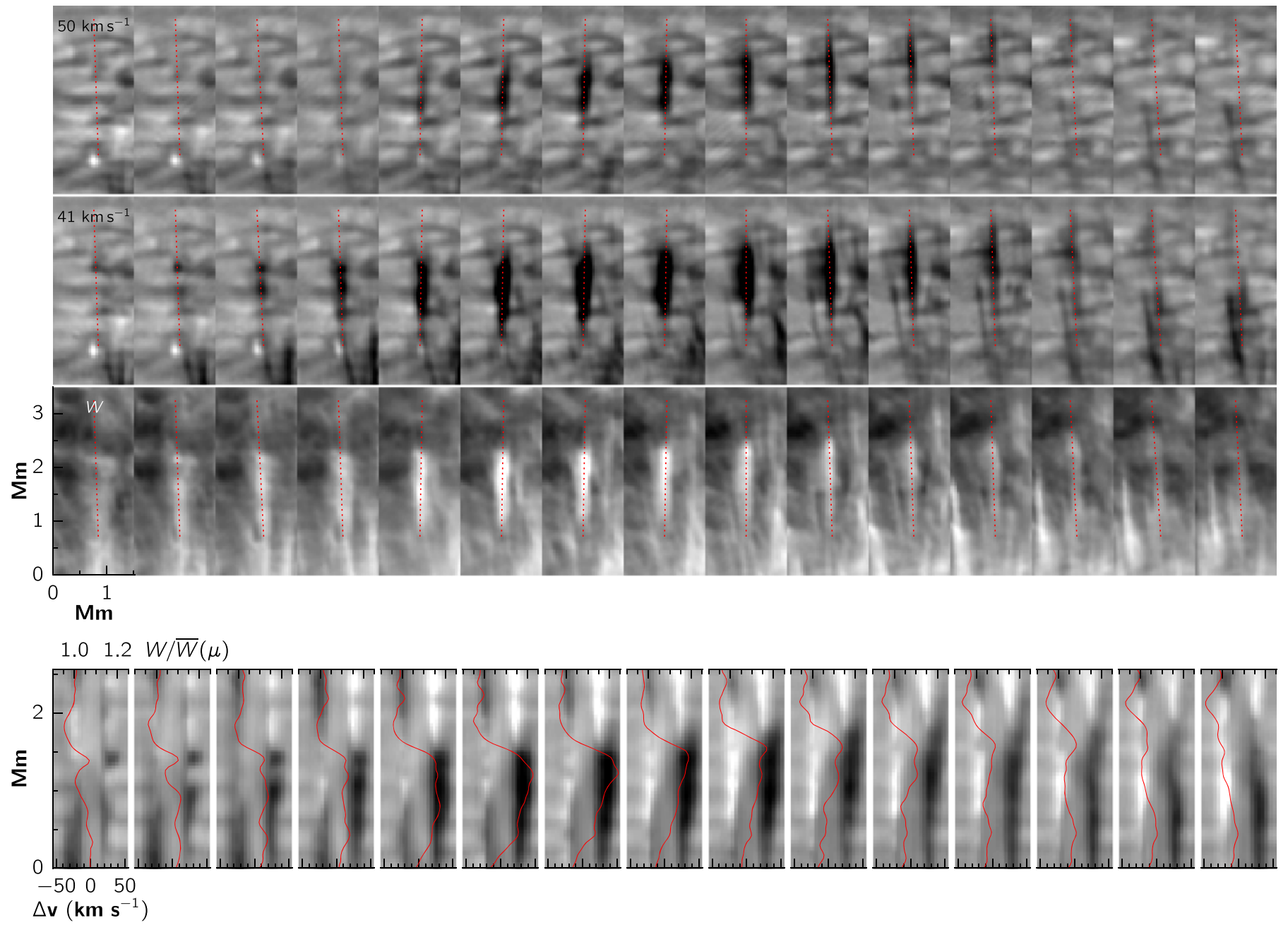

Figure 4. $\mathrm{H} \alpha$ observations of a spicule/fibril on disk. The top three rows show, from top to bottom, the time evolution in the $\mathrm{H} \alpha$ red wing intensities at $50 \mathrm{~km} \mathrm{~s}{ }^{-1}$ $(0.11 \mathrm{~nm})$ from line center, $41 \mathrm{~km} \mathrm{~s}^{-1}(0.09 \mathrm{~nm})$ from line center, and $W$, the FWHM of H $\alpha$ (see text). The spatial size of this small region is $1.5 \times 3.5 \mathrm{Mm}{ }^{2}$. The dotted red lines are cuts along the spicule axis, along which spectrograms are shown in the bottom row. The spectrograms have been divided by the mean H $\alpha$ line profile, therefore black and white represent regions with lower and higher intensity than the mean. Superimposed over the spectrograms are plots of the line width divided by the mean width, with the scale on top (major tickmarks at 1 and 1.2). Time increases from left to right, and the images are shown at the observational cadence of $5.5 \mathrm{~s}$, as noted at the top.

features are visible, and spicules evolve in the same way. This being a quiet Sun region, the overwhelming majority of spicules are type II spicules, and they fade from both filtergrams at the same time. This is important for reconciling the results from Hinode with earlier work on spicules, which was mostly done using $\mathrm{H} \alpha$ observations. In the post-Hinode literature there are very few studies of type II spicules observed with $\mathrm{H} \alpha$. Pasachoff et al. (2009) most likely observed type II spicules in $\mathrm{H} \alpha$, but arrived at the puzzling conclusion that the majority of their spicules were not type I because more than $70 \%$ faded and did not descend; but they were also not type II spicules, because their upward apparent velocities were too low (close to the canonical value of $25 \mathrm{~km} \mathrm{~s}^{-1}$, e.g., Beckers 1968). However, with a cadence of $\approx 50 \mathrm{~s}$, their study misses the detailed dynamics and is most likely affected by spicule confusion due to superposition, which Pereira et al. (2013) found to underestimate the apparent velocities and overestimate the lifetimes. In addition, Pasachoff et al. (2009) measure the apparent velocity as the mean velocity of the spicule in a number of frames, whereas De Pontieu et al. (2007) and Pereira et al. (2012) measure the upward velocity as the maximum (or starting) velocity. Combining all these factors, one suspects that the velocities of Pasachoff et al. (2009) can probably be reconciled with typical velocities of type II spicules $\left(30-110 \mathrm{~km} \mathrm{~s}^{-1}\right)$, and that the authors did observe type II spicules in $\mathrm{H} \alpha$. This would confirm our findings that in $\mathrm{H} \alpha$, type II spicules fade at the end of their lives, just like when observed in $\mathrm{Ca}$ II $\mathrm{H}$ filters.

Spicules become more abundant and densely packed when observed at wavelengths closer to the line core of $\mathrm{H} \alpha$. The appearance of absorption (or emission off the limb) in the wings of $\mathrm{H} \alpha$ is caused by both Doppler shifts and enhanced line widths (LJKG14 estimate that similar magnitude changes in either will change the wing intensities to a comparable degree). Therefore, the decrease in spicule numbers when observing away from the line core to the wings reflects the distributions of line shifts and widths; only the most extreme events are seen in the outer wings. In narrowband filter images away from the core $\left(|\Delta v| \gtrsim 30 \mathrm{~km} \mathrm{~s}^{-1}\right)$ spicules stand out very clearly against a mostly photospheric background, so such 

$11.1 \mathrm{~s}$
$22.1 \mathrm{~s}$
$33.2 \mathrm{~s}$
$44.3 \mathrm{~s}$
$55.3 \mathrm{~s}$
$66.4 \mathrm{~s}$
$77.4 \mathrm{~s}$
$88.5 \mathrm{~s}$
$99.6 \mathrm{~s}$
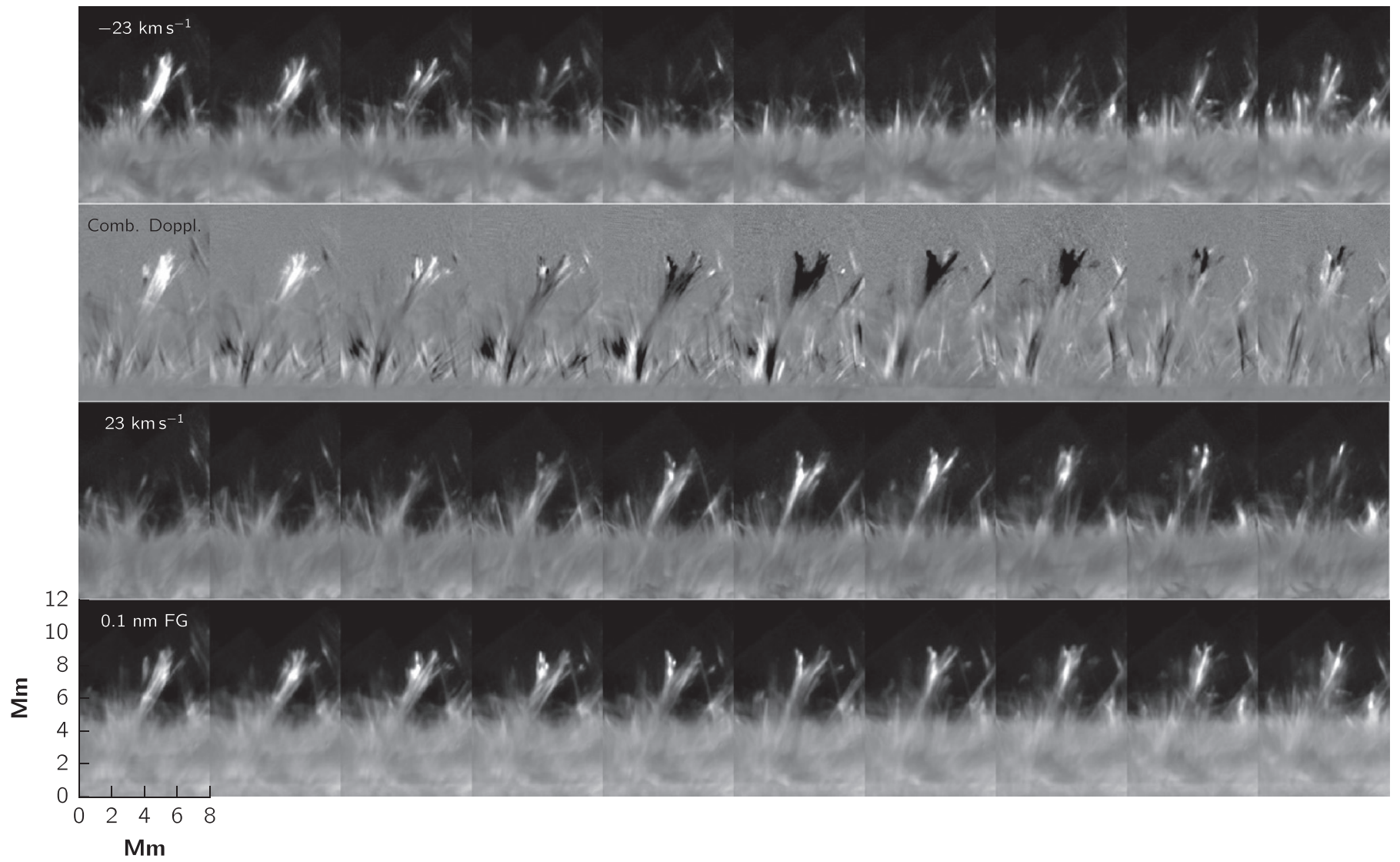

Figure 5. $\mathrm{H} \alpha$ observations of spicules at the limb. From top to bottom, panels show the time evolution in the $\mathrm{H} \alpha$ blue wing intensities at $-23 \mathrm{~km} \mathrm{~s}{ }^{-1}$ ( $-0.05 \mathrm{~nm}$ ) from line center, the combined Dopplergram (see text), the $\mathrm{H} \alpha$ red wing intensities at $23 \mathrm{~km} \mathrm{~s}^{-1}(0.05 \mathrm{~nm})$ from line center, and a filtergram with a $0.1 \mathrm{~nm}$ Gaussian FWHM. The spatial size of this region is $8 \times 12 \mathrm{Mm}^{2}$. The time increases from left to right, with the time difference from the first frame shown on top. The starting UT time of the sequence is 10:29:53.

images have been extensively used to measure the properties of spicules on disk (e.g., Langangen et al. 2008; Rouppe van der Voort et al. 2009; Sekse et al. 2012; JCR12; Sekse et al. 2013a; LJKG14). However, as shown in Figure 4, the transverse swaying motions can make a spicule appear shorterlived when observed further from the line core. In the example of Figure 4, at $50 \mathrm{~km} \mathrm{~s}^{-1}$ the spicule lives for about $40 \mathrm{~s}$, while when taking the full spectral information into account, the event's lifetime is almost twice as long. The full lifetime is not available from a single narrowband image because of changing Doppler shifts. This provides a natural explanation (already speculated by Sekse et al. 2013a) to the fact that lifetimes for disk spicules measured from narrowband images (e.g., Sekse et al. 2013a) are about half the lifetimes for limb spicules (measured from broadband filtergrams, e.g., Pereira et al. 2012).

We have shown that $\mathrm{H} \alpha$ width maps are much more reliable for studying the dynamics of spicules than narrowband images. In such maps spicules stand above the background as bright features and the full history is seen (i.e., not sensitive to Doppler shift changes). Cauzzi et al. (2009) computed similar width maps (calling them a "core width" because, as in our observations, the full spectral profile was not available) in the network, where some spicules are also seen standing out from the dark background. Increased $\mathrm{H} \alpha$ widths are associated with macroscopic motions, but also to temperature increases (Leenaarts et al. 2012). Applying the relations found by Leenaarts et al. (2012) to estimate the gas temperature from the widths is difficult in this case because we do not observe the whole line profile (our widths are measured at lower than half maximum) and we are not observing at disk center. In any case, assuming that width increases are due to temperature alone, using the relations of Leenaarts et al. (2012) we find that the typical width enhancements in spicules $(1.05 \lesssim W / \bar{W}(\mu) \lesssim 1.2)$ amount to temperature increases of about $500-1500 \mathrm{~K}$ compared to where $\mathrm{H} \alpha$ is formed along lines of sight that do not intersect spicular material. A signature of increased line widths in spicules has also been found in lines formed in higher temperatures, from the upper chromosphere to the transition region (Pereira et al. 2014; Tian et al. 2014; Rouppe van der Voort et al. 2015).

With width maps as a robust method for detecting spicules, we find that virtually all cases of the "suddenly appearing" spicules of JCR12 seen in narrowband images can be explained by Doppler motions. This view is also corroborated by Shetye et al. (2016) using a different set of observations, and was also proposed by Kuridze et al. (2015). JCR12 claim that only an optical superposition effect (i.e., spicules as sheets) can explain these "suddenly appearing" spicules. We find that such events only appear suddenly when one's observations are limited to single wavelength narrowband images-using the complete spectral information or width maps we find that the structure was already there, before suddenly appearing at a particular wavelength. Limb spicules have transverse motions on the 
order of 5-30 $\mathrm{km} \mathrm{s}^{-1}$ (e.g., Pereira et al. 2012). Such motions along the line of sight (because there is no reason to assume it is a preferred direction) are enough to cause the changing Doppler shifts that in some cases make spicules appear and disappear suddenly from a narrowband image, requiring no additional abstraction of spicules as fluted sheets.

\section{CONCLUSIONS}

We make use of a unique set of high spatial and temporal resolution observations in several wavelengths of $\mathrm{H} \alpha$ and $\mathrm{Ca}$ II $\mathrm{H}$ filtergrams, obtained during a period of remarkably good, stable seeing. We observe quiet Sun spicules inside the disk and off the limb, and our main findings can be summarized as follows:

1. Spicules have the same properties in $\mathrm{H} \alpha$ and $\mathrm{Ca}$ II $\mathrm{H}$ filtergrams.

2. Spicules become more abundant in wavelengths closer to the $\mathrm{H} \alpha$ line core, reflecting a distribution of Doppler shifts and widths.

3. Measuring the properties of spicules using "single wavelength" narrowband filtergrams can be misleading because Doppler shifts can make the spicule appear and disappear prematurely.

4. $\mathrm{H} \alpha$ width maps provide a robust way of following the evolution of spicules.

5. The sudden appearance of spicules in narrowband images can be explained by transverse motions along the line of sight; one does not need to evoke the sheet model as suggested by JCR12.

This research was supported by the Research Council of Norway through the grant "Solar Atmospheric Modelling" and by grants of computing time from the Programme for Supercomputing, and by the European Research Council under the European Union's Seventh Framework Programme (FP7/ 2007-2013) / ERC Grant agreement No. 291058. This work has benefited from discussions at the International Space Science Institute (ISSI) meeting on "Heating of the magnetized chromosphere" from 2015 January 5 to 8, where many aspects of this paper were discussed with other colleagues. The Swedish $1 \mathrm{~m}$ Solar Telescope is operated on the island of $\mathrm{La}$ Palma by the Institute for Solar Physics of Stockholm University in the Spanish Observatorio del Roque de los Muchachos of the Instituto de Astrofísica de Canarias. This research has made use of SunPy, an open-source and free community-developed solar data analysis package written in Python (SunPy Community et al. 2015).

\section{REFERENCES}

Beck, C., Rezaei, R., \& Puschmann, K. G. 2013, A\&A, 549, A24

Beckers, J. M. 1968, SoPh, 3, 367
Beckers, J. M. 1972, ARA\&A, 10, 73

Carlsson, M., Hansteen, V. H., de Pontieu, B., et al. 2007, PASJ, 59, S663

Cauzzi, G., Reardon, K., Rutten, R. J., Tritschler, A., \& Uitenbroek, H. 2009, A\&A, 503, 577

de la Cruz Rodríguez, J., Löfdahl, M. G., Sütterlin, P., Hillberg, T., \& Rouppe van der Voort, L. 2015, A\&A, 573, A40

De Pontieu, B., Erdélyi, R., \& James, S. P. 2004, Natur, 430, 536

De Pontieu, B., McIntosh, S., Hansteen, V. H., et al. 2007, PASJ, 59, 655

De Pontieu, B., McIntosh, S. W., Carlsson, M., et al. 2011, Sci, 331, 55

De Pontieu, B., McIntosh, S. W., Hansteen, V. H., \& Schrijver, C. J. 2009, ApJL, 701, L1

De Pontieu, B., Title, A. M., Lemen, J. R., et al. 2014, SoPh, 289, 2733

Grossmann-Doerth, U., \& Schmidt, W. 1992, A\&A, 264, 236

Henriques, V. M. J. 2012, A\&A, 548, A114

Judge, P. G., \& Carlsson, M. 2010, ApJ, 719, 469

Judge, P. G., Reardon, K., \& Cauzzi, G. 2012, ApJL, 755, L11

Judge, P. G., Tritschler, A., \& Chye Low, B. 2011, ApJL, 730, L4

Kuridze, D., Henriques, V., Mathioudakis, M., et al. 2015, ApJ, 802, 26

Langangen, Ø., De Pontieu, B., Carlsson, M., et al. 2008, ApJL, 679, L167

Leenaarts, J., Carlsson, M., \& Rouppe van der Voort, L. 2012, ApJ, 749, 136

Lemen, J. R., Title, A. M., Akin, D. J., et al. 2012, SoPh, 275, 17

Lipartito, I., Judge, P. G., Reardon, K., \& Cauzzi, G. 2014, ApJ, 785, 109

Lippincott, S. L. 1957, SCoA, 2, 15

Nishikawa, T. 1988, PASJ, 40, 613

Pasachoff, J. M., Jacobson, W. A., \& Sterling, A. C. 2009, SoPh, 260, 59

Pereira, T. M. D., De Pontieu, B., \& Carlsson, M. 2012, ApJ, 759, 18

Pereira, T. M. D., De Pontieu, B., \& Carlsson, M. 2013, ApJ, 764, 69

Pereira, T. M. D., De Pontieu, B., Carlsson, M., et al. 2014, ApJL, 792, L15

Pneuman, G. W., \& Kopp, R. A. 1977, A\&A, 55, 305

Pneuman, G. W., \& Kopp, R. A. 1978, SoPh, 57, 49

Roberts, W. O. 1945, ApJ, 101, 136

Rouppe van der Voort, L., De Pontieu, B., Pereira, T. M. D., Carlsson, M., \& Hansteen, V. 2015, ApJL, 799, L3

Rouppe van der Voort, L., Leenaarts, J., de Pontieu, B., Carlsson, M., \& Vissers, G. 2009, ApJ, 705, 272

Rutten, R. J. 2006, in ASP Conf. Ser. 354, Solar MHD Theory and Observations: A High Spatial Resolution Perspective, ed. J. Leibacher, R. F. Stein, \& H. Uitenbroek (San Francisco, CA: ASP), 276

Rutten, R. J. 2012, RSPTA, 370, 3129

Scharmer, G. B., Bjelksjo, K., Korhonen, T. K., Lindberg, B., \& Petterson, B. 2003, Proc. SPIE, 4853, 341

Scharmer, G. B., Narayan, G., Hillberg, T., et al. 2008, ApJL, 689, L69

Sekse, D. H., Rouppe van der Voort, L., \& De Pontieu, B. 2012, ApJ, 752,108

Sekse, D. H., Rouppe van der Voort, L., \& De Pontieu, B. 2013a, ApJ, 764,164

Sekse, D. H., Rouppe van der Voort, L., De Pontieu, B., \& Scullion, E. 2013b, ApJ, 769, 44

Shetye, J., Doyle, J. G., Scullion, E., et al. 2016, A\&A, 589, A3

Skogsrud, H., Rouppe van der Voort, L., De Pontieu, B., \& Pereira, T. M. D. 2015, ApJ, 806, 170

Sterling, A. C. 2000, SoPh, 196, 79

Suematsu, Y., Wang, H., \& Zirin, H. 1995, ApJ, 450, 411

SunPy Community, T., Mumford, S. J., Christie, S., et al. 2015, CS\&D, 8, 014009

Tian, H., DeLuca, E. E., Cranmer, S. R., et al. 2014, Sci, 346, 1255711

Tsiropoula, G., Alissandrakis, C. E., \& Schmieder, B. 1994, A\&A, 290, 285

Tsiropoula, G., Tziotziou, K., Kontogiannis, I., et al. 2012, SSRv, 169, 181

van Noort, M., Rouppe van der Voort, L., \& Löfdahl, M. G. 2005, SoPh, 228, 191

Vissers, G., \& Rouppe van der Voort, L. 2012, ApJ, 750, 22

Withbroe, G. L. 1983, ApJ, 267, 825

Yurchyshyn, V., Abramenko, V., \& Goode, P. 2013, ApJ, 767, 17

Zhang, Y. Z., Shibata, K., Wang, J. X., et al. 2012, ApJ, 750, 16 